\title{
Secular Evolution and the Morphological Transformation of Cluster and Field Galaxies
}

\author{
Xiaolei Zhang ${ }^{1}$ and Ronald J. Buta ${ }^{2}$ \\ ${ }^{1}$ Remote Sensing Division, U.S. Naval Research Lab, \\ 4555 Overlook Ave. SW, Washington, DC 20375, USA \\ email: xiaolei.zhang@nrl.navy.mil \\ ${ }^{2}$ Department Physics and Astronomy, University of Alabama, \\ 514 University Blvd E, Box 870324, Tuscaloosa, AL 35401, USA \\ email: buta@sarah.astr.ua.edu
}

\begin{abstract}
Deep surveys conducted during the past decades have shown that galaxies in the distant universe are generally of more irregular shapes, and are disky in appearance and in their star formation rate, compared to galaxies in similar environments in the nearby universe. Given that the merger rate between $\mathrm{z}=2$ and the local universe is far from adequate to account for this observed morphological transformation rate, an internal mechanism for the morphological transformation of galaxies is to be sought, whose operation can be further aided by environmental factors. The secular evolution mechanism, especially with the discovery of a collisionless dissipation mechanism for stars within the secular evolution paradigm, has provided just such a framework for understanding the morphological evolution of galaxies across the Hubble time. In this paper we will summarize the past theoretical results on the dynamical mechanisms for secular evolution, and highlight new results in the analysis of the observational data, which confirmed that density waves in physical galaxies possess the kind of characteristics which could produce theobserved rates of morphological transformation for both cluster and field galaxies.
\end{abstract}

Keywords. galaxy structure, galaxy dynamics, galaxy evolution

\section{Introduction}

It has been a commonly-held belief that the stellar orbit in a galaxy containing quasistationary density wave patterns does not exhibit secular decay or increase, and there is no wave and disk-star interaction except at the wave-particle resonances (Lynden-Bell \& Kalnajs 1972). Zhang (1996, 1998, 1999) first demonstrated that secular orbital changes of stars across the entire galaxy disk are in fact possible due to a collective instability induced by the density wave patterns. The integral manifestation of this process is an azimuthal phase shift between the potential and density spirals, which results in a secular torque action between the wave pattern and the underlying disk matter. As a result, the matter inside the corotation radius loses angular momentum to the wave secularly, and sinks inward. The wave carries the angular momentum it receives from the inner disk matter to the outer disk and deposits it there, causing the matter in the outer disk to drift further out.

The secular morphological evolution process causes the Hubble type of an average galaxy to evolve from late to early (Zhang 1999). The rate of secular evolution is expected to be faster for cluster galaxies than for isolated field galaxies because it is proportional to the wave amplitude squared and pattern pitch-angle squared (Zhang 1998), and cluster galaxies are found to have large amplitude and open density wave patterns excited through the tidal interactions with neighboring galaxies and with the cluster potential as a whole. 

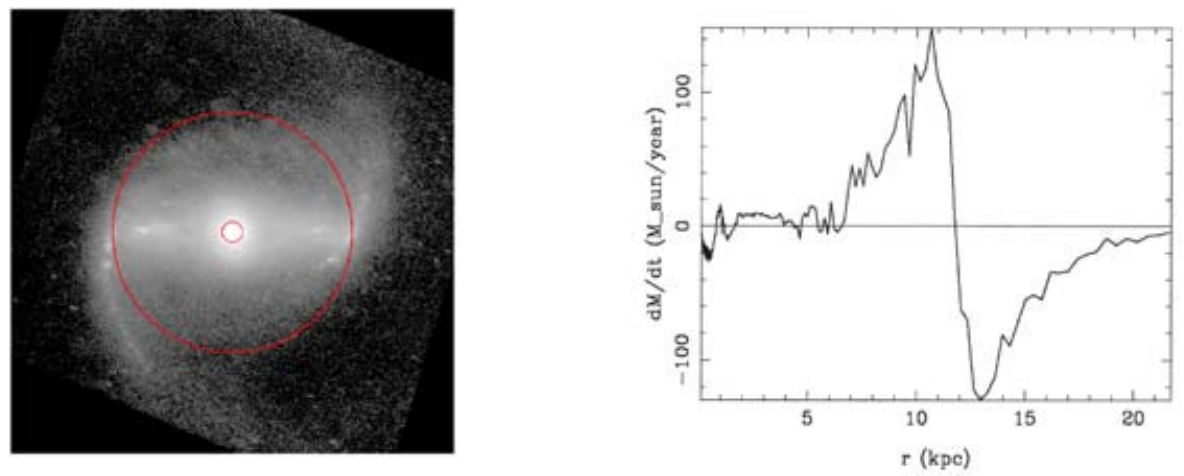

Figure 1. Left: $K_{s}$-band image of NGC 1530 and the superimposed corotation circles determined using the phase shift method (Zhang \& Buta 2006). Right: Radial mass accretion/excretion rate calculated for NGC 1530 from the $K_{s}$-band image.

\section{New Results on the Secular Mass Accretion Rates}

We have found in this study that physical galaxies generally contain density waves strong enough to cause significant mass redistribution over their lifetime. In Figures 2, we show an image of the SBb galaxy NGC 1530 with superimposed corotation circles, and the resulting secular mass accretion/excretion rate calculated using the formulae given in Zhang (1998). The calculated mass accretion/excretion rates for NGC 1530, with peak value around $145 \mathrm{M}_{\odot} /$ year, are several orders of magnitude larger than for our Galaxy $\left(\sim 0.6 \mathrm{M}_{\odot} /\right.$ year; Zhang 1999$)$. This difference is due to the much larger density wave amplitude (average of $70 \%$ ), larger pitch angle $\left(65^{\circ}\right)$ and larger surface density (average of $100 \mathrm{M}_{\odot} / \mathrm{pc}^{2}$ ) of NGC 1530 compared to the Galaxy. We have calculated the same quantities for several other galaxies and obtained more moderate accretion rates, from a few to a few tens of $\mathrm{M}_{\odot} /$ year. In early type galaxies the central mass accretion rate can be much enhanced due to the presence of strong nuclear density wave patterns, even though the outer wave patterns generally become weaker as galaxies age. Thus density-wave induced radial mass accretion could serve as the main driver for the morphological transformation of both the field and cluster galaxies over the past Hubble time, as well as for fueling active galactic nuclei. Furthermore, the mass accretion of the stellar component (in addition to gas) also means that most of the stellar population of the bulge could form long before the shape of the bulge, maintaining a homogeneous and older stellar population of the bulge during the process of secular evolution. This helps to solve one of the main difficulties in the secular building of bulges from the accretion of the gas component alone, which leads to a much varied stellar population in the resulting bulges. The same phase-shift-induced mass redistribution process also works in galaxies which contain other skewed density distributions as observed in many high-z proto galaxies.

XZ acknowledges the support of the Office of Naval Research. RB acknowledges the support of NSF grant AST 050-7140 to the University of Alabama.

\section{References}

Lynden-Bell, D., \& Kalnajs, A.J., 1972, MNRAS, 157, 1.

Zhang, X. 1996, ApJ, 457, 125; 1998 ApJ, 499, 93; 1999 ApJ, 518, 613.

Zhang, X. \& Buta, R.J. 2006, these proceedings. 


\section{Discussion}

Phil Appleton: I would like to ask you about the outflow predicted by the models. Where does the gas end up? Is there evidence of this outflow or pile-up in the galaxies for which your model works?

XIAOLEI ZHANG: The formation of more and more concentrated core as well as a diffused envelope are both predictions of gravitational entropy evolution theory (Lynden-Bell and Wood 1968). Most disk galaxies do have extended outer stellar disks. Part of the outdrifted stars and gas could go back to the intergalactic medium. For nested resonances, even though this implies several hierarchies of inflow/outflow, the dominant trend is found to be a major radial range of inflow, and another major radial range of outflow, mostly in the outer disk with the inner galaxy accretion/excretion rates much smaller than that in the outer galaxy, as shown for NGC 1530. 